Editorials

\title{
Association between Infective Endocarditis and Premalignant Colorectal Lesions
}

\author{
Carles Falces \\ Department of Cardiology, Cardiovascular Institute, Hospital Clínic-IDIBAPS, University of Barcelona, Barcelona, Spain
}

The association between Infective Endocarditis (IE) and Colorectal Cancer (CRC) has been known for more than 50 years. The microorganisms in which this association has been described are Streptococcus gallolyticus (formerly Streptococcus bovis) (Corredoira-Sánchez et al., 2012; Boleij et al., 2011), in different subspecies and, more recently, Enterococcus faecalis (Pericàs et al., 2016; 2017; Escolà-Vergé et al., 2020; Pericàs et al., 2021).

Colonoscopy is the gold standard for the screening and diagnosis of colorectal cancer. American and European IE guidelines recommend the systematic performance of colonoscopy in patients with $S$. gallolyticus bacteremia and high suspicion of IE (Baddour et al., 2015; Habib et al., 2015). Previous studies have shown that colorectal neoplasms in patients with IE due to $S$. gallolyticus are much more frequent than in the general population, so colonoscopy is mandatory (Corredoira-Sánchez et al., 2012). Boleij et al. (2011) systematic review and meta-analysis reported an association between infection from $S$. gallolyticus and CRC in $65 \%$ of cases. Although the association between S. gallolyticus IE and CRC is robust, the association with Colorectal Adenomas (CRA) is less conclusive, because CRA are also very frequent in the general population. Recent studies have shown that, like $S$. gallolyticus IE, E. faecalis IE is associated with Colorectal Neoplasms (CRN) and thus colonoscopy should also be mandatory in these patients (Pericàs et al., 2016; 2017; Escolà-Vergé et al., 2020; Pericàs et al., 2021).

The association between IE ( $S$. gallolyticus or $E$. faecalis) and CRA is highly relevant in clinical practice. In these patients, the diagnosis of severe infectious disease may allow the early diagnosis and treatment of a premalignant colorectal neoplasm.

In the present issue, Vilardell et al. analyze the relationship between streptococcal infective endocarditis and pre-neoplastic colorectal lesions in a retrospective single-center study of a Mediterranean population. In this study, 71 patients with IE due to any microorganism underwent colonoscopy as part of the extension analysis during hospitalization: 49 patients $(69 \%)$ had IE without colorectal lesions, 14 patients (20\%) had IE with dysplastic adenomas and eight $(11 \%)$ had IE with CRC. $S$. gallolyticus was the microorganism most frequently associated with colorectal disease, especially with pre-neoplastic lesions (50\%). Not only high-degree but also low-degree dysplastic adenomas presented this association. The authors conclude that $S$. gallolyticus IE is associated with pre-cancerous colorectal lesions, including low-degree dysplastic adenomas. The results support the current recommendation to perform colonoscopy for the screening of colorectal cancer and pre-neoplastic lesions in patients with $S$. gallolyticus IE.

The relationship between $S$. gallolyticus and colorectal cancer is not well clarified. Colorectal lesions may simply be the gateway for the microorganism, but it may be that it is both a passenger and a cancer-promoting bacterium. For $S$. gallolyticus to facilitate the development of cancer it needs premalignant conditions, like CRA. Therefore, S. gallolyticus is not the main cause of CRC, but an auxiliary factor that accelerates its development (Pasquereau-Kotula et al., 2018).

$S$. gallolyticus is not the only microorganism associated with CRN. Several studies have found that E. faecalis might bear carcinogenetic properties contributing to CRN development. Pericàs et al. (2016; 2017) reported a 17-fold higher prevalence of CRN in patients with E. faecalis IE with an unknown source of the infection undergoing colonoscopy than in the general population. Escolà-Vergé et al. (2020) also found similar rates of $\mathrm{CRN}$ in patients with a known source. More recently, a study from the GAMES cohort added to previous evidence suggesting a much higher rate of CRN among patients with E. faecalis IE than in the general population of the same age and sex (Pericàs et al., 2021).

\section{Conclusion}

The association between $S$. gallolyticus IE and CRN (cancer and pre-malignant lesions) is well established. The indication for colonoscopy in these patients should be mandatory. In light of current data, colonoscopy 
should also be recommended in patients with E. faecalis IE until further studies are available.

\section{References}

Baddour, L. M., Wilson, W. R., Bayer, A. S., Fowler Jr, V. G., Tleyjeh, I. M., Rybak, M. J., ... \& American Heart Association Committee on Rheumatic Fever, Endocarditis and Kawasaki Disease of the Council on Cardiovascular Disease in the Young, Council on Clinical Cardiology, Council on Cardiovascular Surgery and Anesthesia and Stroke Council. (2015). Infective endocarditis in adults: diagnosis, antimicrobial therapy and management of complications: a scientific statement for healthcare professionals from the American Heart Association. Circulation, 132(15), 1435-1486. https://doi.org/10.1161/CIR.0000000000000296

Boleij, A., van Gelder, M. M., Swinkels, D. W., \& Tjalsma, H. (2011). Clinical Importance of Streptococcus gallolyticus infection among colorectal cancer patients: systematic review and meta-analysis. Clinical Infectious Diseases, 53(9), 870-878. https://doi.org/10.1093/cid/cir609

Corredoira-Sánchez, J., García-Garrote, F., Rabuñal, R., López-Roses, L., García-País, M. J., Castro, E., ... \& Varela, J. (2012). Association Between Bacteremia Due to Streptococcus gallolyticus subsp. gallolyticu s (Streptococcus bovis I) and Colorectal Neoplasia: A Case-Control Study. Clinical Infectious Diseases, 55(4), 491-496. https://doi.org/10.1093/cid/cis434

Escolà-Vergé, L., Peghin, M., Givone, F., PérezRodríguez, M. T., Suárez-Varela, M., Meije, Y., ... \& Fernández-Hidalgo, N. (2020). Prevalence of colorectal disease in enterococcus faecalis infective endocarditis: results of an observational multicenter study. Revista Española de Cardiología (English Edition), 73(9), 711-717.

https://doi.org/10.1016/j.recesp.2019.07.016
Habib, G., Lancellotti, P., Antunes, M. J., Bongiorni, M. G., Casalta, J. P., Del Zotti, F., ... \& Zamorano, J. L. (2015). 2015 ESC guidelines for the management of infective endocarditis: the task force for the management of infective endocarditis of the European Society of Cardiology (ESC) endorsed by: European Association for Cardio-Thoracic Surgery (EACTS), the European Association of Nuclear Medicine (EANM). European Heart Journal, 36(44), 3075-3128. https://doi.org/10.1093/eurheartj/ehv319

Pasquereau-Kotula, E., Martins, M., Aymeric, L., \& Dramsi, S. (2018). Significance of Streptococcus gallolyticus subsp. gallolyticus association with colorectal cancer. Frontiers in Microbiology, 9, 614. https://doi.org/10.3389/fmicb.2018.00614

Pericàs, J. M., Ambrosioni, J., Muñoz, P., de Alarcón, A., Kestler, M., Mari-Hualde, A., ... \& Belaustegui, M. C. (2021, January). Prevalence of Colorectal Neoplasms Among Patients With Enterococcus faecalis Endocarditis in the GAMES Cohort (2008-2017). Mayo Clinic Proceedings, 96, 132-146. https://doi.org/10.1016/j.mayocp.2020.06.056

Pericàs, J. M., Corredoira, J., \& Miró Meda, J. M. (2016). Colorectal adenomas. New England Journal of Medicine, 375, 387-390. https://doi.org/10.1056/NEJMc1604867

Pericàs, J. M., Corredoira, J., Moreno, A., García-País, M. J., Falces, C., Rabuñal, R., ... \& Miró, J. M. (2017). Relationship between Enterococcus faecalis infective endocarditis and colorectal neoplasm: preliminary results from a cohort of 154 patients. Revista Española de Cardiología (English Edition), 70(6), 451-458.

https://doi.org/10.1016/j.recesp.2016.09.055 